\title{
A 15 minutos de Los Asperones
}

\author{
15 minutes away from Los Asperones
}

\author{
EUGENIO RIVAS HERENCIA (1) 0000-0003-1771-4467 \\ Universidad de Málaga, Málaga, España.
}

\section{MARÍA RIVAS HERENCIA}

Servicio de Vivienda Protegida de la Junta de Andalucía, Sevilla, España.

Artículo original

Original Article

\section{Resumen}

Correspondencia/

Correspondence

La propuesta $A 15$ minutos de Los Asperones forma parte de un proyecto de investigación multidisciplinar desarrollado en Los Asperones, Málaga. Dos niveles articulan el proyecto: por un lado, se plantea un análisis del territorio y de la realidad sociocultural del barrio en forma de documental que gira en torno a una serie de entrevistas; en segundo lugar, se lleva a cabo una video-performance, una acción poética que se yergue sobre el poder de la utopía y el mito.

A partir de la teoría urbanística de La ciudad del cuarto de hora, que indaga en la idea de ciudad sostenible, se establece la medida espacio-temporal en la que cada ciudadano debería tener acceso, a pie o en bicicleta, a los servicios fundamentales. ¿A qué distancia se encuentran los residentes de Los Asperones de este parámetro? Partiendo del barrio y con un radio de 15 minutos, recorremos esta zona de residuos de la ciudad de Málaga, donde más de 150 familias simulan diariamente el juego del habitar.

PALABRAS CLAVE: caminar, ciudad, 15 minutos, social, Asperones.

Eugenio Rivas

Herencia

eugeniori-

vas@uma.es

Financiación/

Fundings

El proyecto audiovisual $A 15$ minutos de Los Asperones ha sido patrocinado por el Vicerrectorado de Cultura de la UMA dentro de la Convocatoria de proyectos para incentivar la creación artística.

CÓMO CITAR ESTE TRABAJO / HOW TO CITE THIS PAPER

Rivas, E., \& Rivas, M. (2020). A 15 minutos de Los Asperones. Umática. Revista sobre Creación y Análisis de la Imagen, 3 .

Received: 10.08 .2020

Accepted: 28.12 .2020

https://doi.org/10.24310/Umatica.2020.v2i3.11195

Umática. 2020; 3:173-190 


\title{
15 minutes away from Los Asperones
}

\section{Eugenio Rivas Herencia \& MARía Rivas HeREnCIA}

Institución del autor, ciudad, País.

\begin{abstract}
The proposal 15 Minutes away from Los Asperones is part of a multidisciplinary research project developed in Los Asperones, Malaga. Two levels articulate the project: on one hand, an analysis of the territory and the socio-cultural reality of the neighbourhood is proposed in the form of a documentary that revolves around a series of interviews; on the other hand, a video-performance, a poetic action that stands on the power of utopia and myth

Based on the 15 Minutes City theory, which explores the idea of a sustainable city, is established the spatial-temporal measure in which each citizen should have access, on foot or by bicycle, to the fundamental services. How far away are the residents of Los Asperones from this parameter? Starting from the neighborhood and within a radius of 15 minutes, we travel through this waste area of the city of Malaga, where more than 150 families simulate daily the game of living.
\end{abstract}

KEYWORDS: walk, city, 15 minutes, social, Asperones.

\section{Summary - Sumario}
1. Descripción y estado del proyecto
2. Contexto y aparato teórico-conceptual
3. Producción
4. Obra y Resultados 


\section{Descripción y estado del proyecto}

Como introducción a un proyecto más amplio de investigación y transformación del barrio malagueño de Los Asperones, nace la propuesta audiovisual $A 15$ minutos de Los Asperones, actualmente en fase de posproducción. En este trabajo se plantea un análisis del territorio y de la realidad sociocultural del barrio. Después de un periodo inicial de acercamiento y escucha a la realidad de Los Asperones, se trazaron seis recorridos de quince minutos de duración. Los itinerarios querían señalar las seis necesidades básicas de todo ciudadano, establecidas por Carlos Moreno (2020) en su teoría sobre La ciudad del cuarto de hora: alojamiento, trabajo, aprovisionamiento, salud, educación o cultura y ocio. Como primer paso para adentrarnos en la problemática de esta área periférica, realizamos una serie de entrevistas a lo largo de los itinerarios marcados, conversando con diferentes vecinos, agentes del barrio y otros especialistas externos. Paralelamente se llevaría a cabo una acción artística, en la que los autores conducían un carro-mirador por cada uno de los recorridos para concluir en zonas abandonadas en medio de la nada, lugares perdidos en un territorio sin identidad [Fig. 01]. Esta metodología de acercamiento daría lugar a numerosos intercambios con los vecinos del barrio, generando oportunidades de colaboración futura con los involucrados.

Con un fuerte carácter relacional, $A 15$ minutos de Los Asperones condensa la experiencia artística de Eugenio Rivas con las nociones de arquitectura y urbanismo de María Rivas. Tras más de quince años de producción artística, Eugenio dirige su mirada, gracias a la colaboración con María, hacia la repercusión social del proyecto artístico. Lo importante no es la obra, sino el cambio que se produce gracias a ella (Camnitzer, 2020, p. 270). Actualmente, ambos autores continúan trabajando en nuevas propuestas que amplían el estudio y la exploración realizada en Los Asperones, expandiendo su campo de investigación en esa frontera indefinida entre el arte, la arquitectura y la movilización ciudadana. Con A 15 minutos de Los Asperones se abre una línea de trabajo que aspira a la creación colectiva y apuesta por el potencial revolucionario de los pequeños gestos simbólicos.

\section{Contexto y aparato teórico-conceptual}

En 1987 se fundó el barrio de Los Asperones en la periferia de Málaga para alojar temporalmente a más de un centenar de familias de etnia gitana provenientes de diversos asentamientos chabolistas. El desplazamiento desde sus núcleos de origen crearía graves problemas de desarraigo, una situación que con el paso de los años ha desembocado en una insostenible situación de marginalidad y exclusión. En esta barriada, como denuncia Miguel López Melero, «no se cumple ninguno de los derechos humanos. Si repasamos los treinta derechos humanos, yo creo que en Los Asperones no se cumple ninguno [...], ni de la vivienda, ni de la educación, ni de la salud, ni nada» (Rivas y Rivas, 2020). Un alto porcentaje de sus habitantes vive en situación de pobreza extrema y aún hoy, tres décadas más tarde, esta población continúa a la espera de una respuesta acerca de su futuro por parte de las instituciones competentes [Fig. 02]. 
Fig. 01. Eugenio y María Rivas (2020). En torno a Los Asperones. [Serie fotográfica]. Málaga, España.

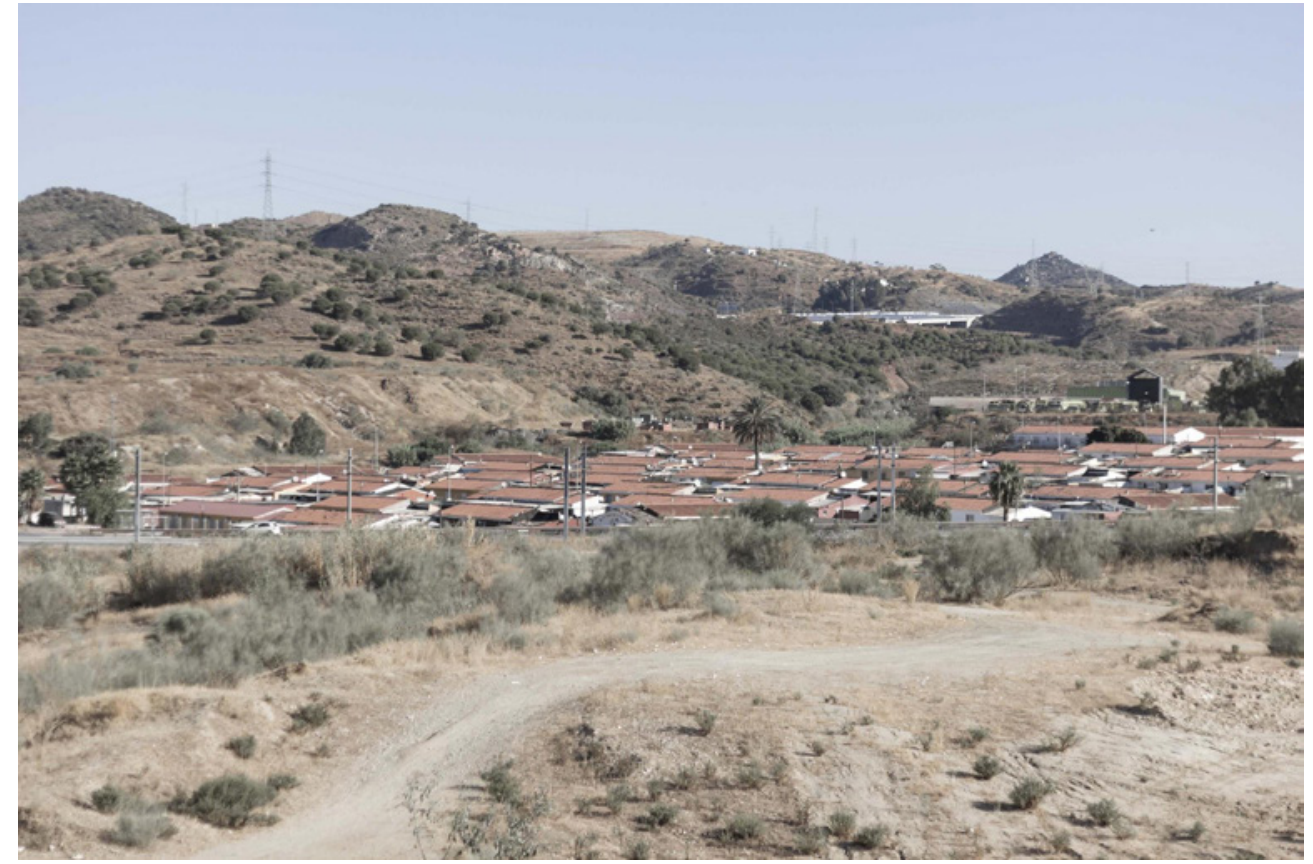

Con el proyecto A 15 minutos de Los Asperones nos acercamos a esta población desplazada de su propia ciudad para analizar los modos en los que el ser humano se ve obligado a relacionarse con su territorio. En este barrio encontramos un claro ejemplo de ese ser arrojado al mundo (Heidegger, 1978), que alertado por la falta de sentido de su existencia se enfrenta a su propio universo y lo mantiene a distancia (Duque, 2008, p. 26). Inspirados por la provocación de los situacionistas, utilizamos la deriva como método de reflexión y experimentación directa de la ciudad (Debord, 1957). Este modo de abordaje artístico radical despojado de toda estrategia que no sea el caminar se mueve con el fin de desorientar el comportamiento y reunir datos de la singularidad del espacio explorado. Esta paradisciplina situacionista de la psicogeografía puede funcionar como una herramienta de investigación del entorno a partir de las emociones y los comportamientos de los individuos (Bishop, 2019, p. 123). Como discípulo actual de Guy Debord y sus compañeros de la Internacional Situacionista, estudiamos las ideas del arquitecto italiano Francesco Careri, quien en su publicación Pasear, detenerse (2016) describe la deriva llevada a cabo junto a su colectivo Stalker a través de espacios estigmatizados o prohibidos en Roma y en diversas ciudades latinoamericanas. Careri reivindica el fuerte potencial del acto de caminar y lo eleva a la categoría de arte. Nos impulsa a la pérdida, al deambular descuidado por la ciudad y sus periferias sin un plan predeterminado. Evoca, así, el descubrimiento más intuitivo y, a la vez, más placentero del territorio. Caminar es precisamente la clave del nuevo paradigma de la ciudad del cuarto de hora. La teoría propuesta por Moreno para la ciudad de París (2020) es heredera del pensamiento promovido por Lefebvre en El Derecho a la Ciudad (2017) y La producción del espacio (2013) y ha sido acogida por otros urbanistas como Dan Luscher en San Francisco (2020). Este modelo urbano cuestiona el modelo de ciudad actual, heredado 


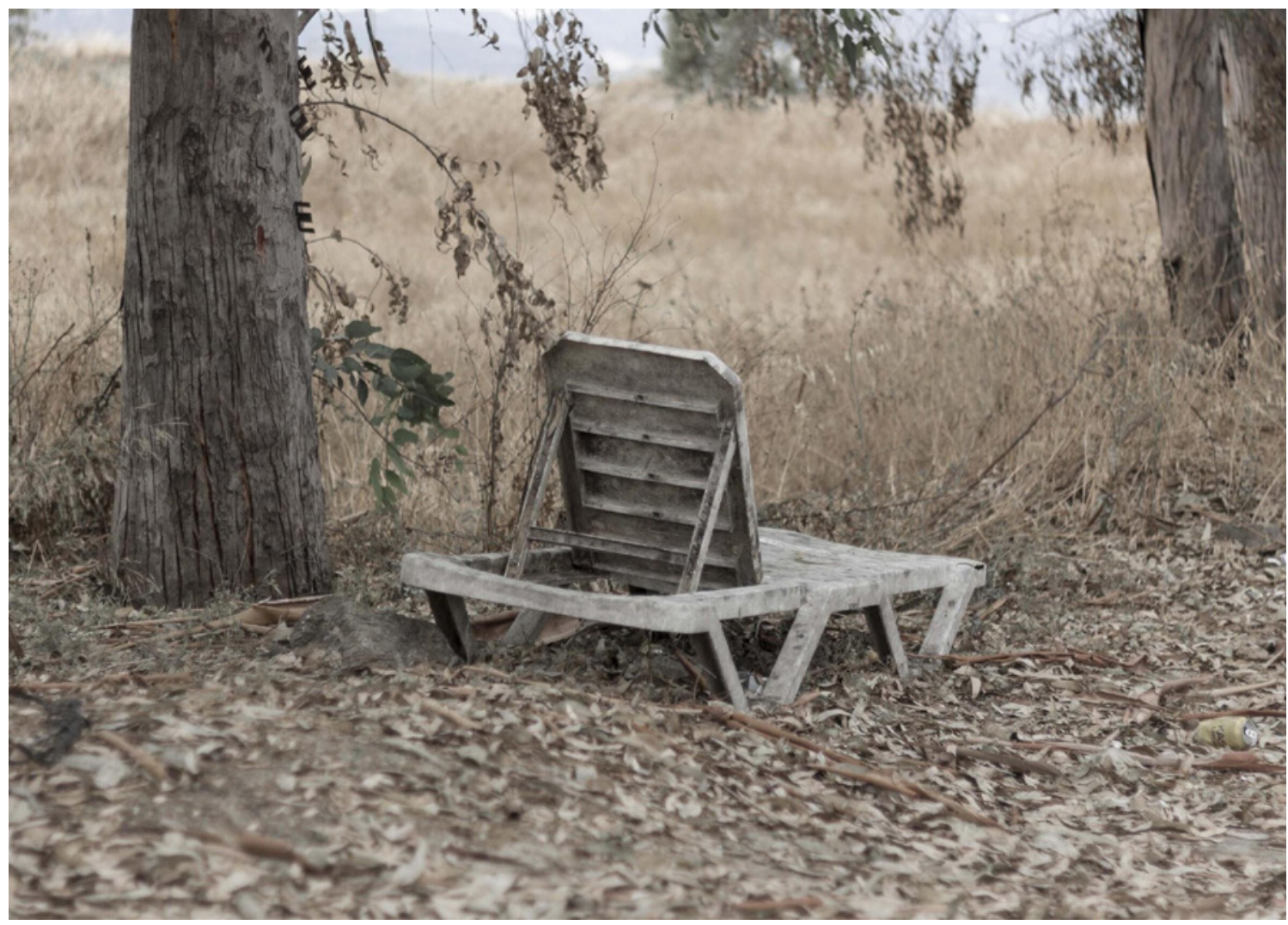

de las teorías urbanísticas centradas en el diseño de la ciudad para el automóvil. En contraposición, este ideal persigue una mayor sostenibilidad, tanto medioambiental como social, y busca aproximar la escala de la ciudad a la dimensión humana. En este sentido, propone la organización de las grandes urbes en torno a múltiples núcleos capaces de satisfacer las necesidades básicas de la población a una distancia máxima de 15 minutos a pie o en bicicleta [Fig. 03]. Este hecho mejoraría la calidad de vida de sus habitantes facilitando su acceso a los mencionados servicios fundamentales. De acuerdo con Moreno «debemos asegurarnos de que todos [...] quienes viven en el centro y quienes viven en la periferia tengan acceso a todos los servicios clave del vecindario» (2020). Y para ello, hemos de apostar por nuevos modelos económicos donde prime el pequeño negocio, donde existan más espacios verdes y donde las infraestructuras previas puedan ser transformadas, reutilizadas y puestas al servicio de la comunidad. En definitiva, se trata de entender la ciudad como un organismo vivo. En este sentido, compartimos el punto de vista de la periodista y activista canadiense Jane Jacobs que a principios de los años sesenta revisa los modelos metropolitanos con su texto Muerte y vida en las grandes ciudades (2011). En una época de grandes reformas urbanísticas en Estados Unidos reivindicaba una mirada más sensible al tejido urbano para poner el acento en las personas, alejándose de las fórmulas racionalistas de tabula rasa que pretendían reconstruir las grandes metrópolis desde cero (Tyrnauer, 2016). La ciudad viva de Jacobs entiende el espacio urbano como un organismo vivo: el espacio
Fig. 02. Eugenio y María Rivas (2020). Entorno a Los Asperones. [Serie fotográfica]. Málaga, España. 


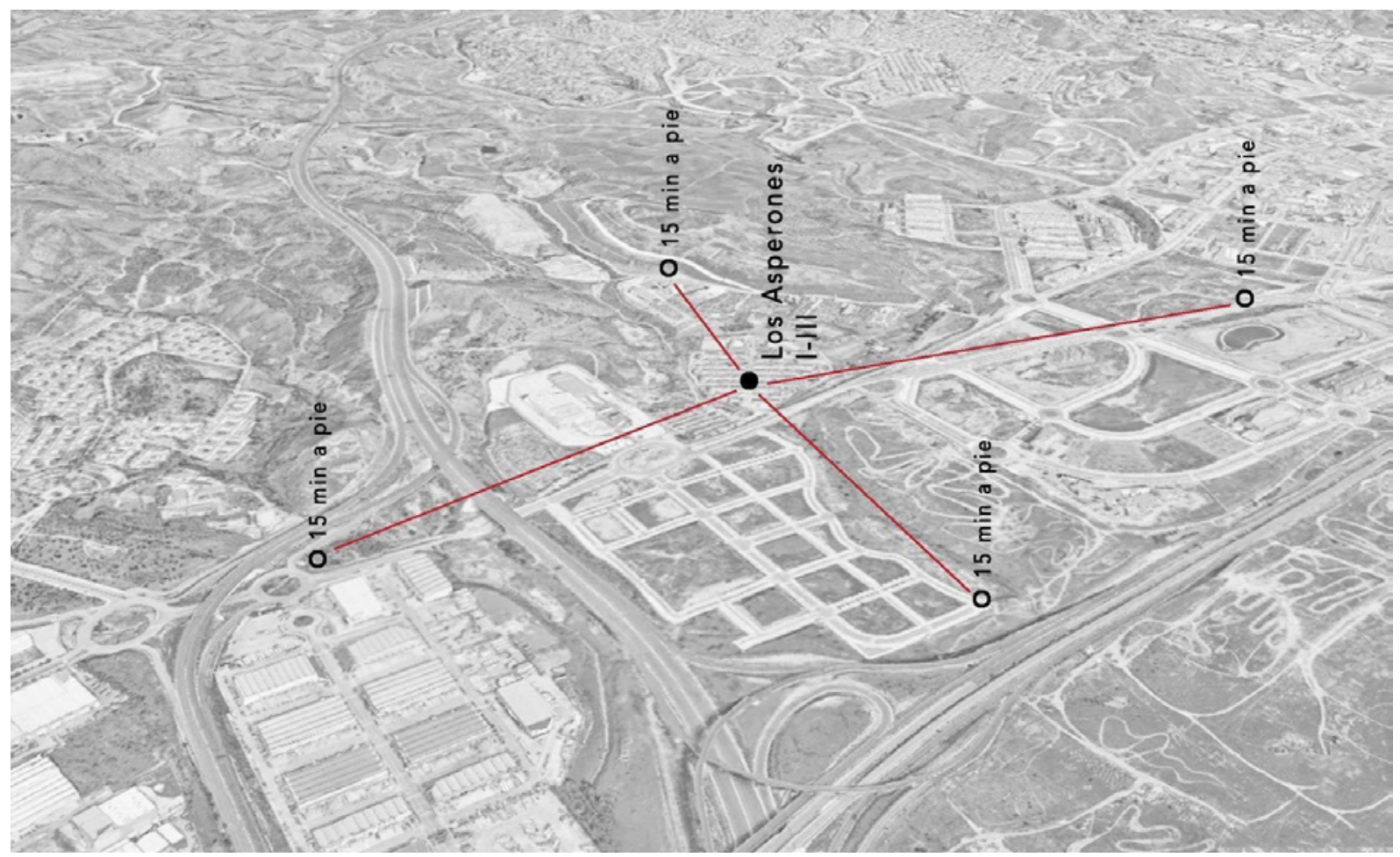

Fig. 03. Diversos puntos accesibles a pie a 15 minutos desde Los Asperones. Imagen de creación propia. público lo conforman las personas en toda su diversidad, la variedad de usos y profesiones, que se dan encuentro en la calle aportando vitalidad al conjunto social y manteniendo a la ciudad viva.

Bajo la mirada de Jacobs es fácil entender la ciudad como un ecosistema dinámico, como el que apunta Pallasmaa en su texto Animales Arquitectos (2020), un ecosistema dignificador para las personas que en ella habitan. Respecto al caso concreto de Los Asperones, Félix de la Iglesia mantiene:

De pronto ellos introducen también una temporalidad [...]. La casa puede ser el soporte, que puede tener carencias, pero, por el contrario, introduce un tiempo que recorre las veinticuatro horas del día, que recorre las estaciones, que sabe cuando está lloviendo y cuando no. Sobre todo porque viven en el territorio, tienen una relación o una dimensión ecológica en las relaciones con el medio tan potentes que la casa no se puede entender sin la componente tiempo. (Rivas y Rivas, 2020)

Tras observar con detalle los modos de habitar de los animales, el arquitecto finlandés señala una curiosa estrategia que consiste en moverse para permanecer (Pallasmaa, 2020, p. 127). Con esta actitud nos proponemos un caminar poético, mezcla de resistencia y aceptación respecto a una realidad sobrevenida para generar disrupción en la conciencia propia del vecindario. Proponemos un cambio de perspectiva que eleve la mirada para ampliar hori- 
zontes y dibujar caminos de fuga, salidas de urgencia que muestren el camino de la dignidad. Una dignidad que solo puede existir de manera compartida (Marina, 1993). Como defiende Jacobs: «Las ciudades tienen la capacidad de proveer algo para cada uno de sus habitantes, sólo porque, y sólo cuando son creadas para todos» (Tyrnauer, 2016). La práctica artística o arquitectónica se integra en las capas más profundas de su contexto sociocultural activando lo que podríamos definir como ecologismo estético (San Martín, 2007), un modo de actuar que promueve la revolución desde el individuo. Esta ecología de la cultura actúa simbólicamente contra la lógica de la realidad y lo predecible para romper los límites del sentido común. Una utopía en movimiento, que en palabras de Eduardo Galeano, se sitúa en un horizonte siempre inalcanzable y alimenta nuestro derecho a soñar con la función última de ponernos en marcha, para hacernos caminar (Barberà, 2015). Como La Nueva Babilonia (2009), concebida por Constant tras su visita en 1956 a la ciudad de zíngaros establecida en la ciudad piamontesa de Alba, Los Asperones es una ciudad lúdica e incierta, una ciudad entrópica que no quiere renunciar a su esencia nómada. Una ciudad móvil anclada en la periferia, pero en continua mutación. Es una ciudad de talla humana que, a pesar de permanecer más de tres décadas en el mismo lugar, no se conforma con quedarse y sigue esperando su momento para echar a rodar. Ante esta situación de emergencia nos animamos a recorrer, documentar, cartografiar e iluminar este escenario invisibilizado en el imaginario común y cuestionar una vez más nuestro modo de estar en el mundo, nuestras maneras de hacer mundos (Goodman, 1978). Manteniendo esa mirada desprejuiciada que nos sugiere Careri (2013), proponemos estos seis recorridos a pie, que deberían facilitarnos el acceso a los servicios fundamentales, satisfacer las necesidades básicas y proveernos, en definitiva, del derecho a la ciudad. Arte y arquitectura entrelazan sus armas en $A_{15}$ minutos de Los Asperones para revisar las constelaciones sociales donde habitamos, entender lo que significa vivir con plena dignidad en la ciudad de hoy y reflexionar sobre cómo creamos y construimos nuestros mundos, tanto desde las decisiones políticas, como desde los hábitos cotidianos del día a día. Mediante un trabajo de escucha generamos experiencias conjuntas y establecemos un plano de reflexión sobre una problemática que desgraciadamente se ve repetida a lo largo de nuestra geografía. La generación de cambio es el objetivo más deseable para esta amplia realidad que, como a Los Asperones, ahoga a numerosos núcleos de población en las sociedades contemporáneas. No podemos olvidar que «en el momento en que un individuo emprende una acción, cualesquiera que fuese, ésta comienza a escapar a sus intenciones» (Morin, 2004, p. 115), pero la transformación debe comenzar por erradicar el analfabetismo, mejorar la empleabilidad, defender la vivienda digna y consolidar el acceso a los servicios básicos. Un cambio que no podrá desarrollarse si no logramos engendrar nuevos sueños y generar una fuente de energía autónoma que empodere a la población y le permita confiar en su propia iniciativa.

En su libro Construir y habitar: Ética para la ciudad, Richard Sennett (2018) diferencia entre dos dimensiones que configuran nuestra realidad urbana: la ciudad de Dios y la ciudad del hombre. Mientras la primera se nutre de significados subjetivos, percepciones, lemas o senti- 
mientos; la segunda se caracteriza por las propiedades más físicas y materiales de nuestras urbes. Frente al mundo de las ideas, esta segunda dimensión contiene la riqueza y la crudeza de lo táctil, una fisicidad que cada habitante goza o sufre en su destinada ciudad. No sabemos cuál de esos dos mundos se encuentra más abandonado o desatendido en Los Asperones, este territorio expulsado del paraíso de la unidad (Sloterdijk, 2020, p. 16-17). Es irremediable admitir que esta población vive lejos de esa tierra idílica del consenso y la unanimidad entre las personas. Sin embargo, la riqueza simbólica y el imaginario de su población son factores incuestionables que juegan a su favor. Ante la terrible pobreza de recursos materiales, es la dimensión más onírica la que puede entablar la salvación, la que debe ser reforzada en primer plano y desde la que debemos partir. Bajo este convencimiento establecemos el tono del presente trabajo. Entre la delgada línea que separa la realidad de la leyenda y el mito nos lanzamos al territorio como traperos de la iconografía (Bourriaud, 2015, p. 92) para desordenar lo preestablecido y hacer crujir la estructura de valores que lo soporta. Los vecinos de esta área, de etnia gitana, poseen su propia cultura y formas de expresión. Su carácter nómada originario se refleja en la inexistente diferenciación entre espacio público y privado. Y existe una percepción de la comunidad como un todo, una gran familia. En este contexto, el caminar sin destino, con la utopía en el horizonte, pretende señalar la ruta de salida para una situación de aislamiento y desesperación. Como confesara Rollo May: «no me importa quién haga las leyes de una sociedad mientras yo pueda crear los mitos» (1998, p. 58).

\section{Producción}

Eugenio y María Rivas han estado a cargo de la dirección y producción del proyecto. La documentación audiovisual del conjunto de entrevistas ha sido desarrollada por la productora Lenonfilms: con fotografía y vídeo a cargo de Lourdes Rodríguez y María Giner; con René Varón encargado del manejo y grabación con dron; y con Emilio J. Fernández como responsable de sonido, edición y producción [Fig. 04]. Por otro lado, la documentación y edición de la acción artística correría a cargo de Lucas Alcántara en colaboración con Manuel Rocha.

El trabajo A 15 minutos de Los Asperones se estructura en dos niveles. Una primera capa atiende a la necesidad de acercarse a la realidad física y socio-cultural del barrio. Este bloque del proyecto se desarrollaría alrededor de una serie de visitas y paseos por el entorno con el ánimo de comprender con mayor profundidad la problemática particular del lugar. Para llevar a cabo dicha aproximación nos sumamos a la red de trabajo ya existente, en la que participan diferentes organizaciones sin ánimo de lucro junto con los servicios sociales del Ayuntamiento de Málaga y la inestimable labor abordada desde el CEIP María de la O. Este bloque culmina con un conjunto de seis entrevistas en las que los autores recorren el territorio junto a diferentes vecinos, agentes involucrados en el contexto y especialistas en diferentes materias. Cada conversación sigue uno de los seis recorridos trazados para salir del barrio en busca de las necesidades fundamentales [Fig. 05]. En una segunda capa se lleva a cabo una acción poética en la que los autores se turnan para desplazar un dispositivo fabri- 


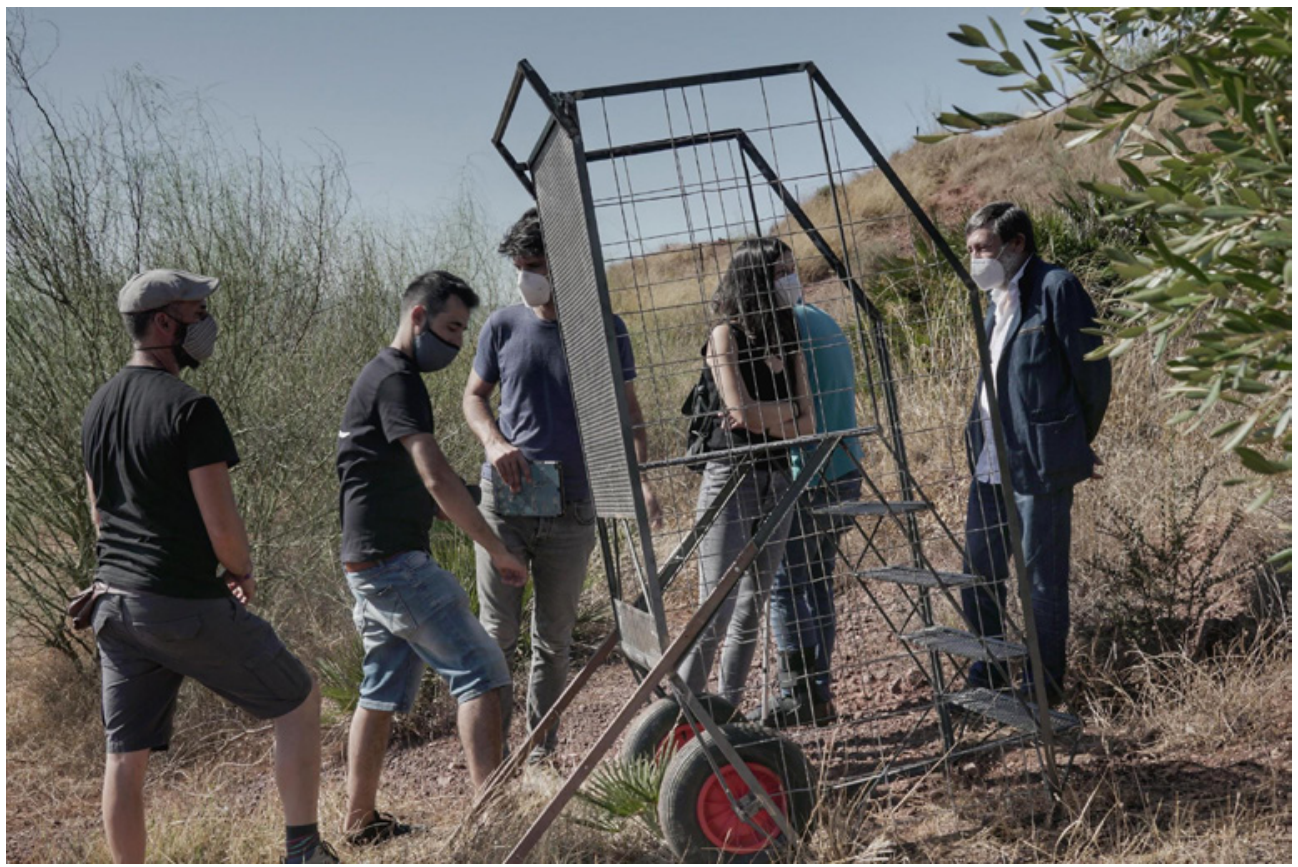

Fig. 04. Eugenio y María Rivas (2020). A 15 minutos de Los Asperones. [Proceso de grabación]. Málaga: Lenonfilms. Fotografía de María Giner.

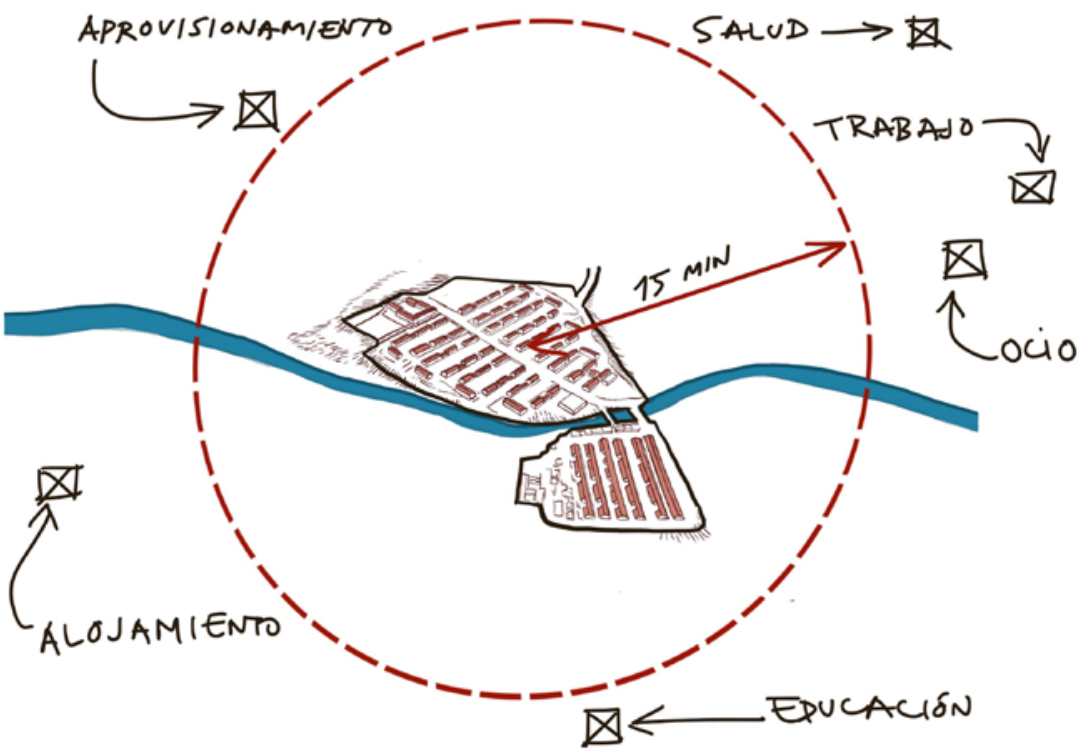

Fig. 05. Eugenio y María Rivas (2020). Mapa A 15 minutos de Los Asperones. Imagen de creación propia.

cado exprofeso que transportan por las mismas rutas. El carro-mirador construido con restos de diversos perfiles metálicos recupera la idea del nomadismo propia de la etnia gitana e incorpora la idea de elevación característica de las torres de defensa o de los miradores turísticos para proponer un cambio de perspectiva [Fig. 06]. Como el Sísifo de Camus (2006), los autores empujan su artefacto simbólico dibujando imposibles salidas sobre un territorio circundante al barrio que, al ser enfrentado desde la escala 1:1, la escala de lo real, impone sus propias medidas físicas y temporales. 
Fig. 06. Eugenio y María Rivas (2020).

A 15 minutos de Los Asperones. Acción. [Fotograma del vídeo]. Málaga: Lucas Alcántara.

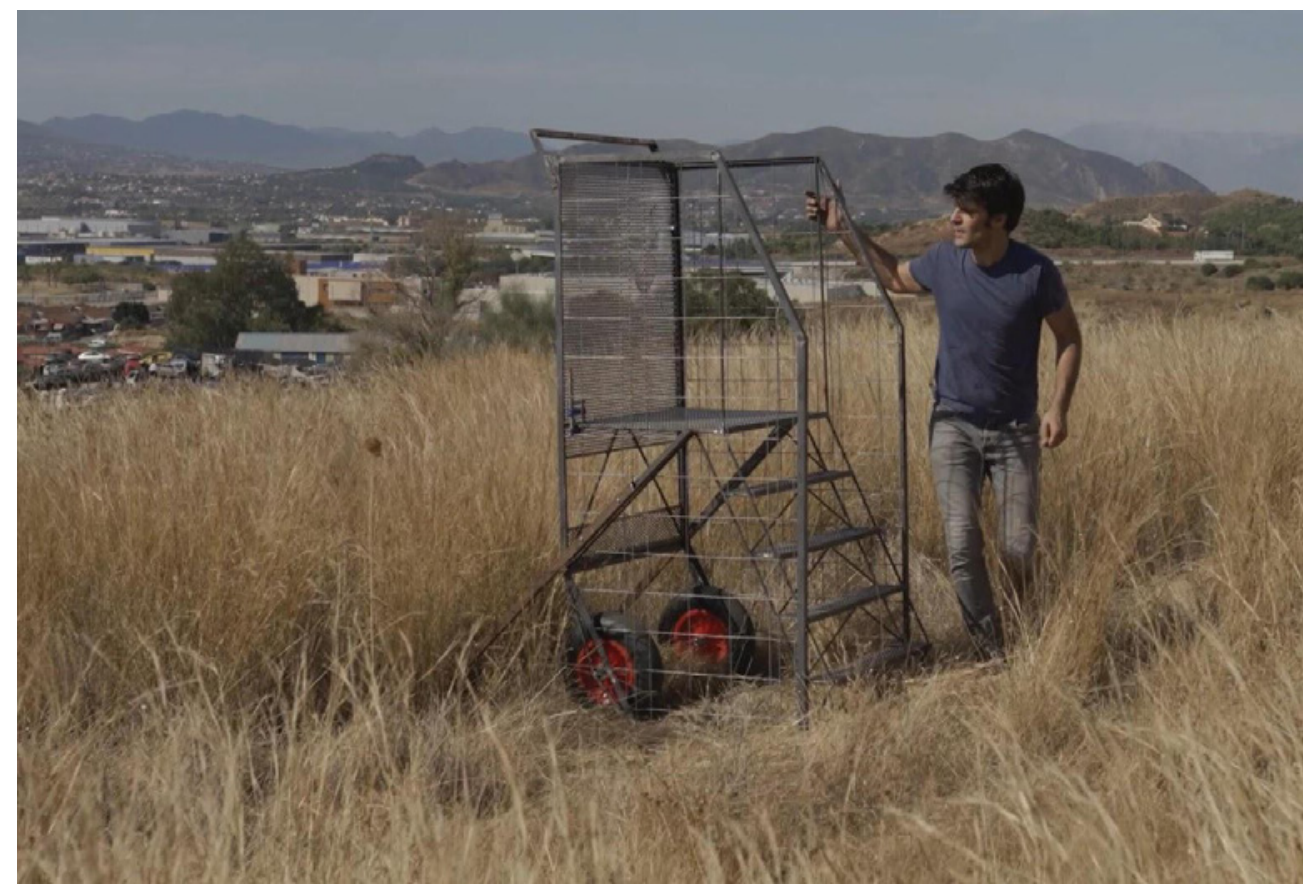

Luis Camnitzer hace distinción entre la capacidad disruptiva del Art Thinking (Acaso, 2017, p. 17) y el carácter predecible del pensamiento científico cuantitativo: desde el territorio de la creatividad hemos aprendido a aceptar lo imposible como parte de la realidad. Mediante la incorporación de lo impredecible, promovemos un modo de construcción de conocimiento basado en la creación de experiencias sociales y modelos de acción, donde cada proyecto configura su propia utopía de proximidad (Bourriaud, 2013, p. 8). De acuerdo con López Melero, los habitantes de Los Asperones «Tienen que tomar conciencia de que su única manera es la cultura y la educación y tienen, no que integrarse en la sociedad hegemónica, sino hacer crujir a la sociedad hegemónica y que respeten a su cultura» (Rivas y Rivas, 2020). Si «las fronteras son instrumentos de fragmentación, separan en lugar de unir, y sirven para definir recipientes cerrados en lugar de crear campos de conexiones» (Camnitzer, 2020, p. 273), la obra de arte relacional debe establecer un contexto simbólico de interacción entre el creador y su ámbito social, cultural y político. Hablamos de un espacio ampliado de intercambio en la frontera desde el que invertir el carácter antipedagógico de la separación, desde el que ocuparse de lo desatendido, de lo marginal, hasta lograr el acuerdo entre todos los involucrados y, al fin, la integración de la diversidad.

Caminando desde el barrio hacia sus alrededores en trayectos de quince minutos se mantuvieron seis conversaciones principales, como apuntábamos anteriormente. En ellas, además de asignar a cada participante una de las necesidades o servicios básicos de la ciudad, se establecieron temas generales de reflexión sobre la estructura social, sus valores y fortalezas, así como la situación de exclusión y posibilidades de cara a un futuro inminente. Numerosas conversaciones con los vecinos fueron documentadas y conforman un material de gran interés para los proyectos que en la actualidad se siguen desarrollando y en los 
que se persigue incorporar una mayor participación de la comunidad. En este sentido, y para adaptarnos al plan de trabajo establecido, fueron seleccionadas seis de las conversaciones donde la mayor parte de los contenidos consiste en las aportaciones de especialistas en diversas materias ligados en mayor o menor medida al barrio o a contextos similares. Con ello abrimos la visión subjetiva a una perspectiva plural que pretende seguir creciendo a medida en que continuamos profundizando en las particularidades del barrio y en las múltiples subjetividades que en él se condensan.

Con el arquitecto y profesor en la Escuela Técnica Superior de Arquitectura de la Universidad de Sevilla, Félix de la Iglesia Salgado, se realizaría el contexto de Los Asperones en su conjunto, la ciudad de Málaga como telón de fondo y el mar en el horizonte. Este trayecto nos conducía hasta un punto elevado sobre el barrio con la idea de asumir un cambio de perspectiva. Desde esta altura era posible visualizar el contexto en su conjunto, con la ciudad de fondo y el mar en el horizonte. Respecto a la dignidad de la vivien$\mathrm{da}$, de la Iglesia nos remite a modelos estereotipados que garantizan las aspiraciones de muchos, pero nos advierte que hemos de entender la dignidad como «algo mucho más relacionado y vinculado con el usuario, con el sitio, con la comunidad en la que vive» (Rivas y Rivas, 2020). De lo que concluye que es preferible hablar de un soporte habitacional capaz de posibilitar los modos de vida singulares, así como los comportamientos que se derivan de estas visiones subjetivas [Fig. 07]. Trazamos el itinerario de acceso al trabajo junto a José María Alonso Calero, profesor de Bellas Artes y hasta 2020 Vicerrector Adjunto de Innovación Social y Emprendimiento en la Universidad de Málaga. Reflexionamos sobre el estigma que supone la pertenencia al barrio a nivel cultural y laboral, así como sobre la necesidad de renovar los símbolos que refuercen la identidad colectiva en base a referentes cercanos. En un tercer recorrido partimos del barrio con Enrique Larive López, miembro de lacasavacía y profesor en la Escuela Técnica Superior de Arquitectura de la Universidad de Sevilla, y Ma Victoria Segura Raya, miembro de lacasavacía y arquitecta en IDE, Gerencia de Urbanismo del Ayuntamiento de Sevilla, para revisar la noción de aprovisionamiento [Fig. 08]. Entre otros aspectos, destacamos la condición aislada de este núcleo habitacional que podría equipararse a la de un campo de refugiados por la falta de infraestructuras. Esta herida abierta en el territorio, como define Larive, «genera finalmente una especie de zuncho habitado [...], un espacio totalmente acotado y cerrado y que impide mecanismos de relación» (Rivas y Rivas, 2020), lo que hace de Los Asperones un lugar completamente insalubre, si lo analizamos desde el punto de vista ambiental. Junto con Francisco Marín Fernández, vecino del barrio, caminamos en dirección a la estación de metro, ya que para acceder a los servicios de salud las distancias han de salvarse mediante un transporte público de difícil acceso y baja frecuencia. Atravesamos espacios vacíos, lugares inexistentes en la estructura de la ciudad, pero que afectan físicamente a estos ciudadanos y establecen una separación entre el barrio y su mundo. En el quinto trayecto conversamos sobre educación y cultura con Miguel López Melero, catedrático emérito de Didáctica y Organización Escolar en la Universidad de Málaga y Francisco Javier Velasco Fano, director del CEIP 
María de la O. Partimos sobre la idea de inclusión educativa para concluir que resulta contradictorio plantear este propósito en un barrio situado en los límites de la ciudad, junto al basurero y al cementerio municipal. Como defiende López Melero: «La única manera para hablar de inclusión sería haciendo disolver o haciendo desaparecer el barrio con todo lo que hay ahí, incluso el propio colegio» (Rivas y Rivas, 2020). Por lo que la sociedad malagueña debe tomar conciencia hasta conseguir que estas personas se incorporen libremente en los diferentes barrios de la ciudad como unos ciudadanos y unas ciudadanas más. Para cerrar esta serie de caminatas temáticas, analizamos las opciones de ocio y divertimento junto a Álvaro Carrillo Eguilaz, arquitecto y profesor colaborador honorario en la Escuela Técnica Superior de Arquitectura de la Universidad de Málaga. A partir del ejemplo que supone el colegio para el barrio, debatimos sobre la posibilidad de aprovechar los espacios públicos fuera del horario institucional para otros usos que atiendan a las necesidades de la comunidad. Este recorrido nos conduce, a través de unas pistas de arena, hasta un gran montículo de un circuito improvisado de cross que separa la visión de Los Asperones de la ampliación del campus de Teatinos de la Universidad malagueña y que evidencia la falta de voluntad para vincular este territorio con el resto de la ciudad.

Fig. 07. Eugenio y

María Rivas (2020)

A 15 minutos de Los Asperones.

Entrevistas.

[Proyecto audiovisual, 30 minutos]. Málaga:

Lenonfilms.

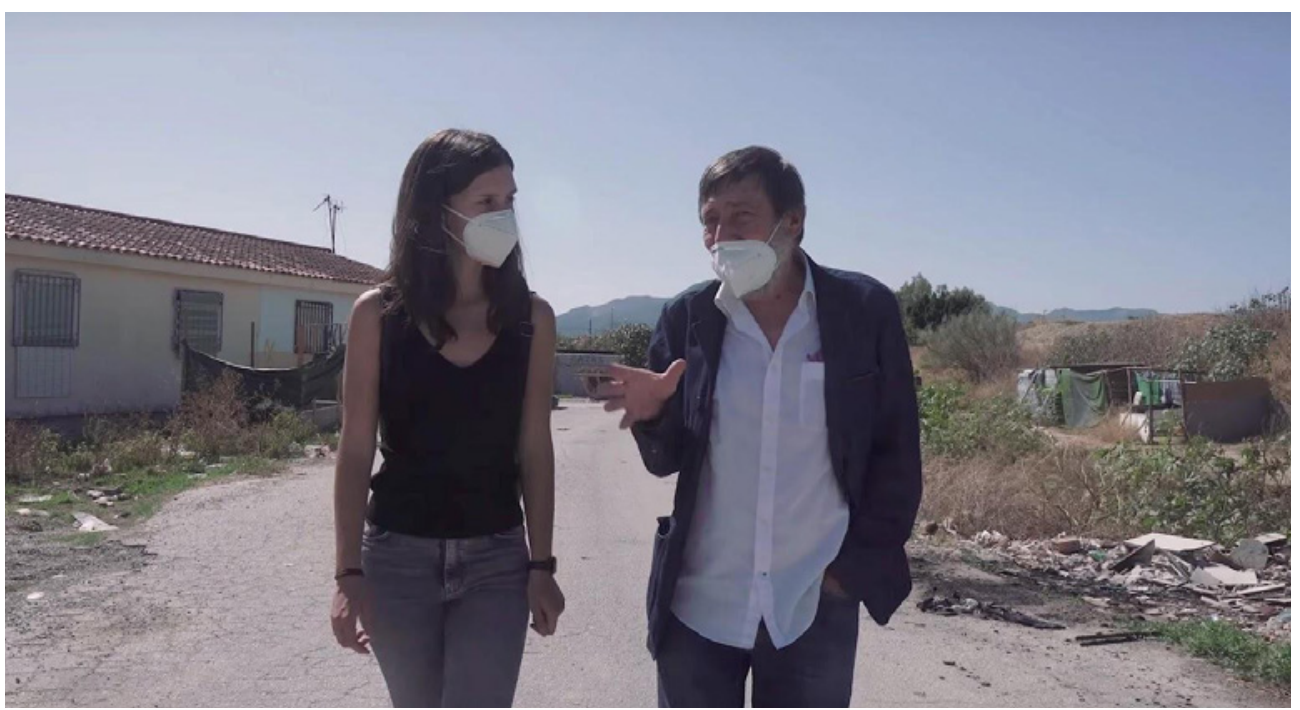

Paralelamente a las entrevistas se registraron numerosas conversaciones con diferentes vecinos, siempre abiertos a compartir sus experiencias y expectativas, así como con otras personas involucradas en Los Asperones. Este material ha sido empleado para otros proyectos como En el corazón de Los Asperones (en colaboración con Lucas Alcántara) galardonado con el premio del jurado en el concurso Entreplanos de la Fundación Arquitectura Contemporánea, Córdoba, 2020 [Fig. 09]. En la actualidad, y partir de las conclusiones aportadas en este primer encuentro con la realidad del territorio, los autores siguen trabajando en varios proyectos enfocados a la participación ciudadana como mecanismo impulsor del cambio. 


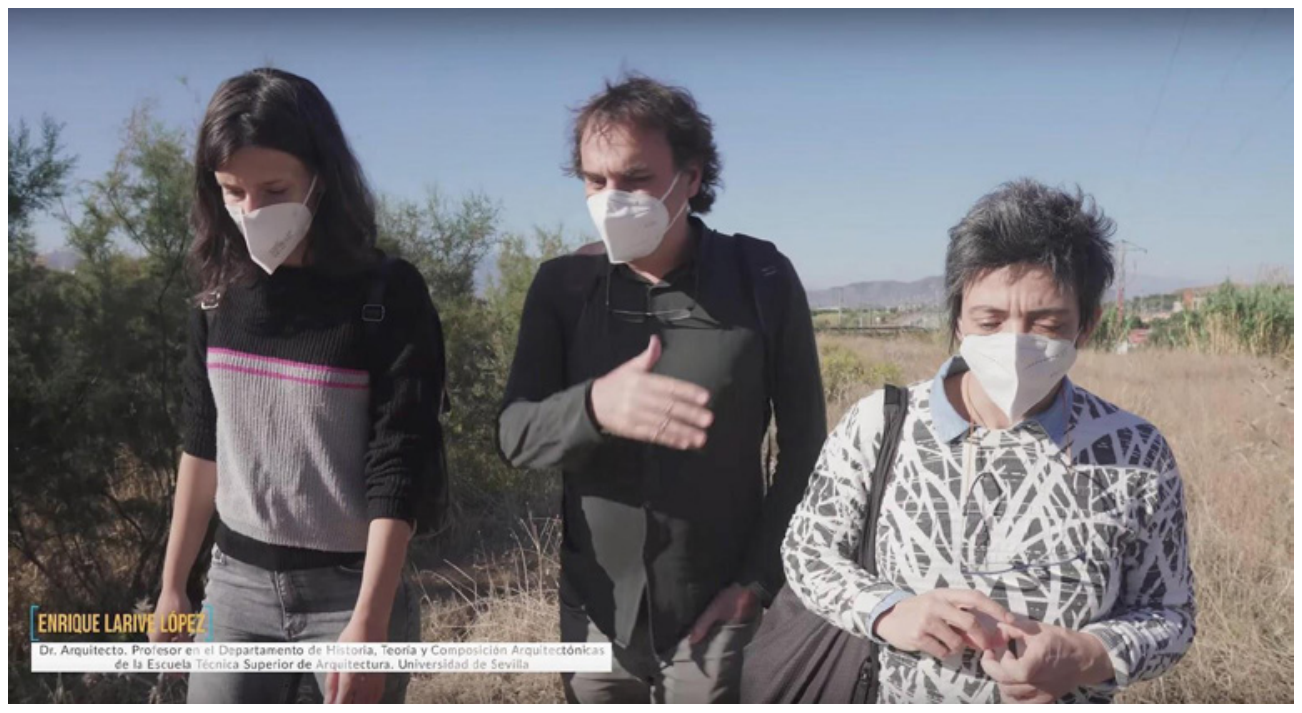

Fig. 08. Eugenio y María Rivas (2020). A 15 minutos de Los Asperones. Entrevistas. [Fotograma del vídeo]. Málaga: Lenonfilms.

Fig. og. Eugenio y María Rivas y Lucas Alcántara (2020). En el corazón de Los Asperones.

[Proyecto audiovisual, 2 minutos. Primer premio Entreplanos]. Córdoba, Fundación Arquitectura Contemporánea.

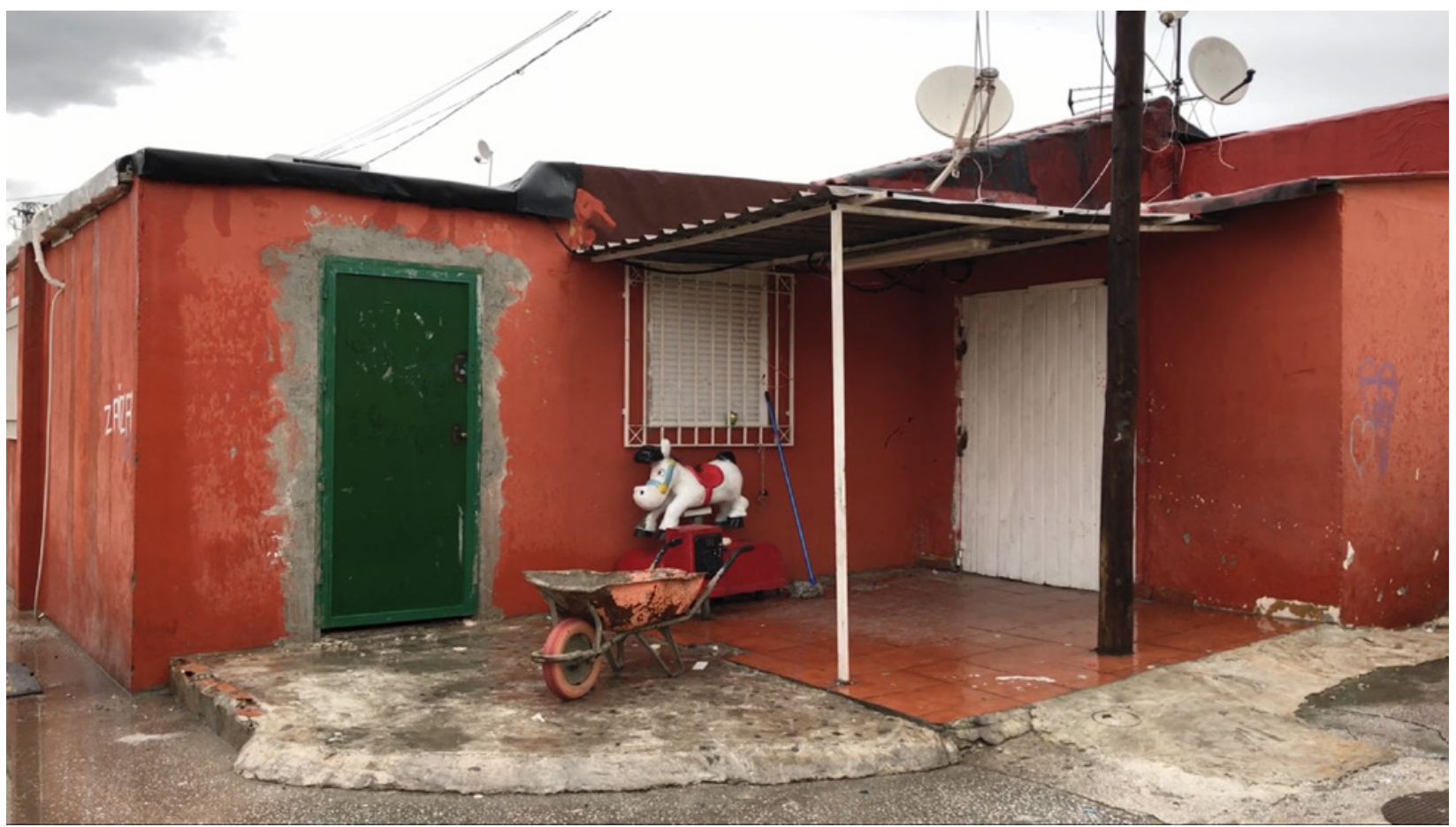

Según lo previsto, el plan de trabajo se desarrollaría en tres fases generales de preproducción, rodaje y postproducción. En la preproducción se llevó a cabo una investigación previa entre los meses de junio y septiembre de 2020. Durante este periodo se realizaron numerosas visitas al barrio, se establecieron diferentes reuniones con agentes sociales y educativos, así como con una gran cantidad de vecinos interesados en prestar sus experiencias para la reflexión y el planteamiento del proyecto. En base a los objetivos previos, la grabación sería planificada tras la exploración del territorio y sus alrededores. Las diferentes sesio- 


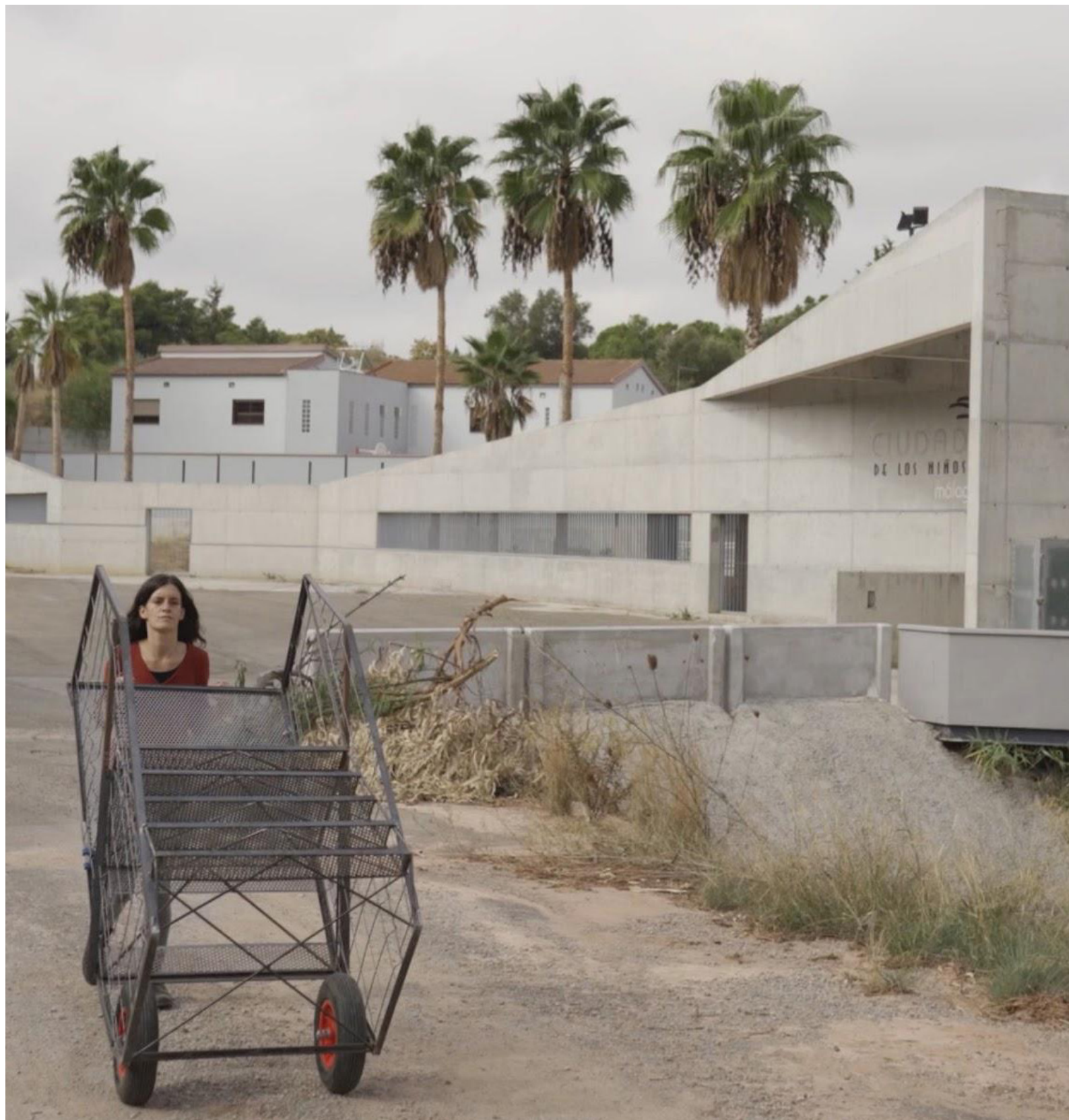

Fig. 10. Eugenio y María Rivas (2020).

A 15 minutos de

Los Asperones. Acción. [Fotograma del vídeo. Detalle].

Málaga: Lucas Alcántara. nes de rodaje se llevaron a cabo entre los meses de septiembre y octubre. Dentro de la postproducción se realizó un primer corte del proyecto para finales del mes de octubre de 2020. Posteriormente se ha seguido trabajando en la edición del trabajo final con repetidas sesiones de visualización, revisiones y correcciones hasta la elaboración de un montaje definitivo [Figs. 10 y 11]. Está prevista una primera proyección en el barrio de Los Asperones antes de verano de 2021, a modo de presentación previa al estreno oficial a cargo del Vicerrectorado de Cultura de la Universidad de Málaga. Este evento quiere sumarse a otras acciones en el territorio con el ánimo de recuperar la identidad colectiva y fomentar la participación vecinal. 


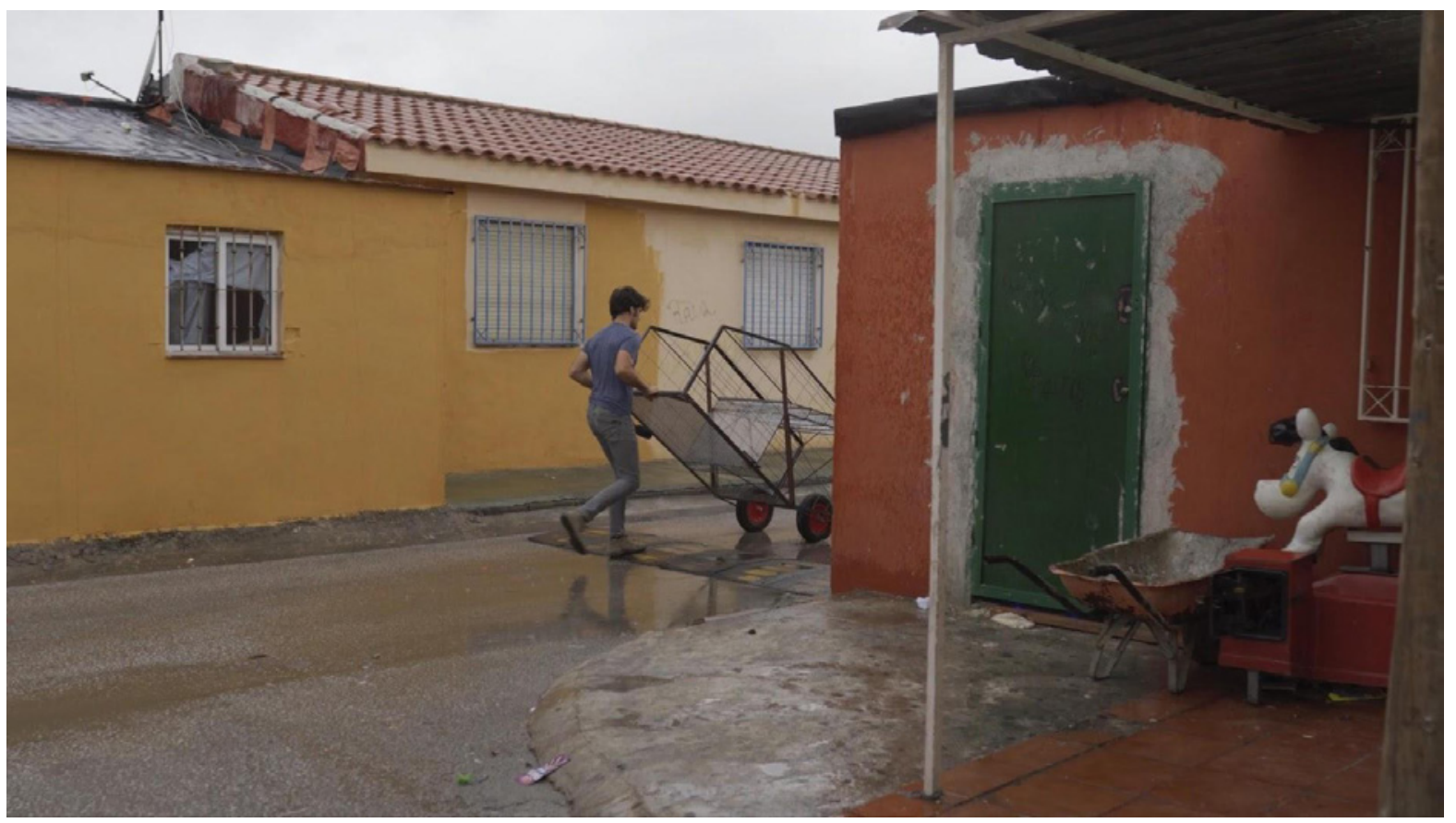

\section{Obra y Resultados}

Como alarma López Melero, «las 295 familias, los hombres y mujeres, los niños y niñas que viven en ese barrio, son considerados, como diría Bauman, residuos humanos» (Rivas y Rivas, 2020). Esto hace prácticamente imposible hablar de inclusión y nos deja el gran desafío de la disolución completa del barrio, dejando que cada uno de sus vecinos y vecinas ejerza la libertad de incorporarse a la pluralidad de la sociedad malagueña. En este sentido, es mucho lo que el pueblo gitano puede aportar a los actuales modelos sociales y a su creciente diversidad. Que esta cultura defienda su propia idiosincrasia y valores no implica una falta de interés por la pertenencia al conjunto social. Hemos de abandonar el concepto neoliberal de integración, que supone la indiscutible aceptación de las normas impuestas por la mayoría hegemónica, para defender el derecho a la diferencia y la diversidad mediante la incorporación de las minorías (Frigiliana, 2020, p. 59). A partir de las conclusiones arrojadas por la experiencia desarrollada con este proyecto se han trazado diferentes estrategias de acción que persiguen la mayor involucración de los vecinos en la toma de decisiones y el desarrollo de los futuros proyectos. Asimismo, estamos multiplicando el número de agentes externos implicados, además de reconducir las estrategias de intervención hacia un modelo de trabajo cooperativo.

Entendemos el proyecto $A 15$ minutos de Los Asperones como un primer paso en el acercamiento a la comunidad [Fig. 12] y la antesala de otros trabajos en los que se incorporen iniciativas plurales. En él se mezclan visiones internas y personales con perspectivas externas de carácter profesional. En un siguiente paso aspiramos hacia un modelo de cooperación en el que la autoría individual pueda disolverse para facilitar la creatividad del colectivo. De esta
Fig. 11 Eugenio y María Rivas (2020). A 15 minutos de Los Asperones. Acción. [Fotograma del vídeo]. Málaga: Lucas Alcántara. 
manera, en las siguientes intervenciones con el vecindario apuntamos a la creación compartida como medio para, en palabras de Bishop, desmaterializar la obra de arte en un proceso social (2019, p. 42). Para ello, planteamos la generación de un espacio que refuerce la identidad grupal en el vecindario, donde la comunidad pueda ejercer una mayor influencia en los proyectos desarrollados. En la actualidad estamos trabajando en diferentes líneas metodológicas participativas que pretenden reforzar la autonomía de los ciudadanos y ciudadanas de Los Asperones y, en definitiva, ayudarles a convertirse en los verdaderos protagonistas del cambio en su contexto.

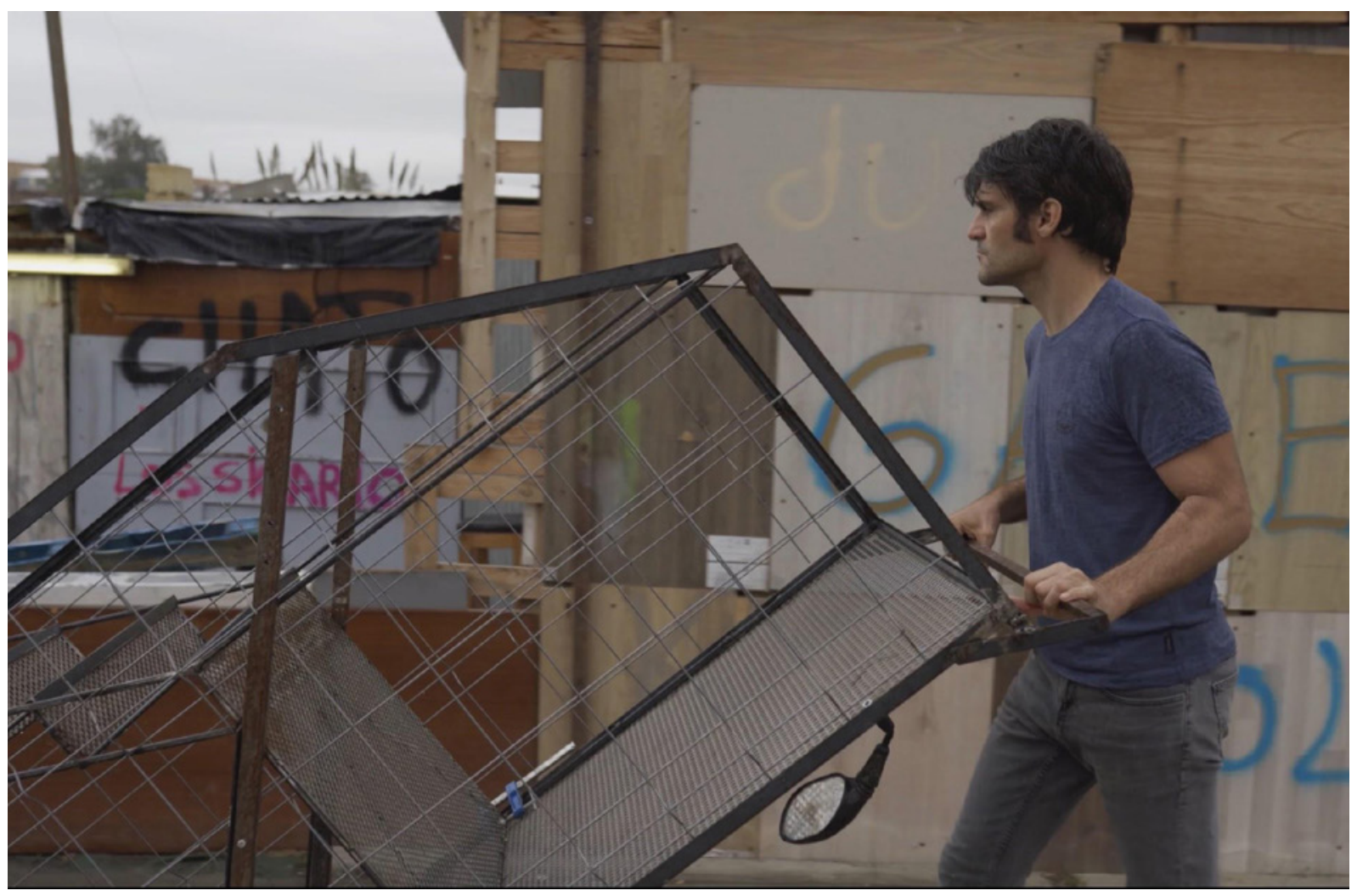

Fig. 12. Eugenio y María Rivas (2020).

A 15 minutos de Los Asperones. Acción. [Fotograma del vídeo]. Málaga: Lucas Alcántara.
Como conclusión general del proyecto subrayamos la dicotomía entre cuidar o desmantelar este particular barrio. Si desde un inicio éramos capaces de intuir esta contradicción, a medida que profundizábamos en la problemática hemos podido comprender que ambas acciones son igualmente necesarias y complementarias. La urgencia por el cuidado de las personas y la lucha por el fin de una estructura precaria, donde hombres y mujeres, niños y niñas, sufren cada día la despreocupación de sus iguales, han de darse la mano en un ejercicio simbólico y político. El activismo y la poesía pueden fundirse en un ejercicio que no se rinda a una solución unidireccional y que, gracias a una multiplicidad de medidas menores, acabe funcionando como el mito para calar en la estructura de valores de nuestra sociedad. Una vibración que consiga establecer nuevos órdenes que afecten no solo a las 
personas que sufren esta situación o situaciones similares, sino a todos los individuos que conforman nuestro mundo, a todos y todas las que ponemos a prueba cada día las incontables maneras de construir el mundo.

\section{Agradecimientos}

El proyecto A 15 minutos de Los Asperones ha sido posible gracias al patrocinio del Vicerrectorado de Cultura de la Universidad de Málaga. Los autores del proyecto agradecen por su colaboración y asesoramiento a La Asociación Chavorrillos, a los Servicios Sociales Puerto de la Torre del Ayuntamiento de Málaga, al personal del CEIP María de la O y a INCIDE Málaga. Queremos mostrar nuestra gratitud en reconocimiento al fuerte compromiso de los entrevistados: Álvaro Carrillo Eguilaz, Enrique Larive López, Mạ Victoria Segura Raya, Félix de la Iglesia Salgado, José Manuel Santiago, Francisco Javier Velasco Fano, Miguel López Melero, Francisco Marín Fernández "Moreno" y José María Alonso Calero. Asimismo, expresamos un agradecimiento especial a todos los vecinos de Los Asperones por su lección de resistencia y de vida.

\section{Referencias}

ACASO, M. y MEGÍAS, C. (2017). Art Thinking. Cómo el arte puede transformar la educación. Madrid: Paidós.

BISHOP, C. (2019). Infiernos artificiales. Arte participativo y políticas de la espectaduría. Ciudad de México: Taller de Ediciones Económicas.

BOURRIAUD, N. (2008 [1998]). Estética relacional. Buenos Aires: Adriana Hidalgo.

BouRRIAUD, N. (2015). La exforma. Buenos Aires: Adriana Hidalgo.

FELIU, M. (productora ejecutiva) Singulars. Barcelona: Sala i Martí.

https://www.ccma.cat/tv3/alacarta/singulars/eduardo-galeano/video/3541530/

CAMNITZER, L. (2020). Manual Anarquista de Preparación Artística. DATJornal, 5(2). 267-274.

CAMUS, A. (2006 [1942]). El mito de Sísifo. Madrid: Anaya.

CARERI, F. (2013). Walkscapes. Barcelona: Gustavo Gili.

CARERI, F. (2016). Pasear, detenerse. Barcelona: Gustavo Gili.

CONSTANT (2009). La Nueva Babilonia. Barcelona: Gustavo Gili.

DEBORD, G. (1958). Teoría de la deriva. en el 2 de Internationale Situationniste. Traducción extraída de (1999). Internacional situacionista, vol. I: La realización del arte. Madrid: Literatura Gris.

DUQUE, F. (2008). Habitar la tierra. Madrid: Abada Editores.

GoODMAN, N. (1978). Maneras de hacer mundos. Madrid: Visor.

FILIGRANA, P. (2020). El pueblo gitano contra el sistema-mundo. Reflexiones desde una militancia feminista y anticapitalista. Ciudad de México: Akal. 
JACOBS, J. (2011 [1961]). Muerte y vida de las grandes ciudades. Madrid: Capitán Swing.

LEFEBVRE, H. (2017 [1968]). El Derecho a la Ciudad. Madrid: Capitán Swing.

LEFEBVRE, H. (2013 [1974]). La producción del espacio. Madrid: Capitán Swing.

LUSCHER, D. (2020). The 15-Minute City Project. San Francisco. https://www.15minutecity.com/

MARINA, J. A. (1993). Teoría de la inteligencia creadora. Barcelona: Anagrama.

MORENO, C. (2020). La ciudad del cuarto de hora. [TedTalks, Octubre 2020]. Francia: TED.

https://www.ted.com/talks/carlos_moreno_the_15_minute_city/transcript?language=es

MAY, R. (1998). La necesidad del mito. La influencia de los modelos culturales en el mundo contemporáneo. Barcelona: Paidós.

MORIN, E. (2004). Introducción al pensamiento complejo. Barcelona: Gedisa.

PALLASMAA, J. (2020). Animales arquitectos. Barcelona: Gustavo Gili.

PUJOL, G. (redacción) y BARBERÀ, J. (director). (2015). Eduardo Galeano. (Temporada 2015, Episodio 14 de abril). [Programa de tvz CCMA]. En Feliu, M. (producción). Singulars. Corporació Catalana de Mitjans Audiovisuals, SA

RIVAS, E., y RIVAS, M. (2020). A 15 minutos de Los Asperones. Entrevistas. [Proyecto Audiovisual]. Málaga: Vicerrectorado de Cultura de la Universidad de Málaga.

SAN MARTín, F. J. (2007). Una estética sostenible. Arte en el final del Estado del bienestar. Pamplona: Cátedra Jorge Oteiza, Universidad de Navarra.

SENNETT, R. (2018). Construir y habitar. Ética para la ciudad. Barcelona: Anagrama.

SLOTERDIJK, P. (2020 [1993]). En el mismo barco. Madrid: Siruela.

TYRNAUER, M. (director) (2016). Citizen Jane: Batalla por la ciudad. [Documental]. Estados Unidos: Altimeter Films. 